\title{
Thermoelectric Cooling in Downhole Measuring Tools
}

\author{
Rohitha Weerasinghe \\ University of the West of England, Bristol, UK
}

\begin{abstract}
Thermoelectric cooling is a highly effective and a novel technique used for cooling solid state circuitry inside downhole measuring equipment. A Peltier device in a deep well measuring tool works as a heat pump. The understanding of the thermal behaviour inside is important in designing the tools. For this study, thermal modelling is used, but accurate modelling of the Peltier device is crucial in predicting the thermal behaviour and subsequent thermal management of the tool. Present paper is based on numerical simulations using computation fluid dynamics and experimental analysis of the thermal behaviour inside an industry standard deep well measuring tool based on a model based Peltier cooling device. Present modelling results are based on two mathematical models to predict the thermal behaviour of the Peltier device. Results show a techniques that can be used in designing cooling performance of downhole equipment and generally in Peltier cooling at elevated temperatures.
\end{abstract}

\section{INTRODUCTION}

\subsection{Downhole tooling}

Vertical seismic profiling (VSP) is a common geophysical technology used in oil and gas investigation in deep wells. VSP provides high resolution and dependable results but is subjected to high temperatures and harsh well environments. As these wells become deeper and hotter, the need for effective thermal management in the instrumentation becomes paramount in keeping the electronics of the measuring tool within allowable working temperatures. Seismic surveys are a critical element of the search for oil and gas exploration. In a seismic survey, the propagation of elastic waves through the rock gives an indication of the sub surface distribution of different rock types and thus the probability of finding a viable source of hydrocarbons. The seismic source varies depending on the application but may be dynamite, an air gun, or a vibrating plate to produce waves at a range of frequencies. In a surface seismic study usually both the source and the receivers are located at the surface and the reflected waves are analysed. These sensitive ground velocity sensors are called geophones. In a Vertical Seismic Profiling borehole investigation the receivers are located within the borehole and the source is usually located at the surface or, less frequently, downhole. Another important and fast growing application for borehole seismic logging tools is the monitoring of hydraulic fracturing (fracking) sites.

\subsection{The cooling problem}

Instrumentation used in borehole seismic investigations needs to be extremely sensitive in order to capture the micro-seismic waves at the receiver and large volumes of data must be sent back to the surface-in the case of continuous monitoring surveys this must be in near real time. This process requires sensitive and sophisticated electronics to be exposed to extremely hostile environments; depending on the wellbeing surveyed this may be pressures of up to $30,000 \mathrm{psi}(2000 \mathrm{bar}$ or $206 \mathrm{MPa})$, temperatures of greater than $200{ }^{\circ} \mathrm{C}$ and often in environments with high concentrations of $\mathrm{H}_{2} \mathrm{~S}$. The electronics manufacturers are continually pushing to increase the service temperatures of their products. Various strategies have been deployed to achieve this over the last 30 years including vacuum (Dewar) insulation, eutectic alloys which extend the time available downhole and various active cooling technologies as evidenced by Bennett et.al. (1986). Unlike in conventional electronics cooling applications, the need for the system to be hermetically sealed and withstand high pressures prevents the use of forced air cooling. In addition to shielding the electronics from the heat of the borehole fluid, a further challenge is in dissipating the heat produced by the electronics themselves. Various active cooling techniques have been evaluated in the past. 


\section{PROBLEM DEFINITION}

\subsection{Peltier cooling for downhole tools}

Thermoelectric cooling has been around since the early 1950s and extensive research has taken place in aid of cooling at room temperature and around. The phenomenon that a voltage is generated in a conductor or semi conductor subject to a temperature gradient was discovered around 1800 and is known as the Seebeck effect. The inverse process where heat is pumped across a conductor or a semi-conductor due to a voltage difference is known as the Peltier effect. The current uses of the Seebeck effect are mainly for thermoelectric heat recovery. Thermoelectric cooling is used in applications where space is limited and where conventional refrigerants cannot be used, for example in computers and small machinery. Most these applications happen around ambient temperature. Manufacturers of Peltier conductors have tested their devices at room temperature and within a band of $30 \mathrm{~K}$ either side of that. Seismic down-hole measuring devices operate under harsh environmental conditions, a few kilometres below ground at temperatures that are $200 \mathrm{~K}$ above standard room temperature. These tools contain sensitive electronic circuitry and the performance of the devices depend on whether these devices can be kept within the operating temperatures of the electronics. A lot of studies have taken place in thermoelectric heat recovery at elevated temperatures and representative data are available. However, the availability of representative data in cooling at these temperatures are rare. As a representative technique according to Dirker et.al. (2005), the properties are being extrapolated from room temperature results. Thermoelectric cooling in seismic measuring tools is a novel technology. The performance of such devices has been hampered by the relatively low overall coefficient of performance. However, this low COP has been resulted mostly by the losses in the thermal passage of such devices. Thermal analysis has shown that the performance can be enhanced by better insulation the device and carefully arranging the thermal paths. Heat transfer modelling has help improve this aspect of the measuring tools. Thermal analysis of Peltier performance can be used to analyse the overall device performance by identifying the heat transfer patterns, paths and the actual cooling perfomance of the Peltier device. The present study looks at the performance of the Peltier devices in cooling at elevated temperatures and the validity of heat transfer models used to predict the properties.

\subsection{Thermoelectric Devices.}

The amount of heating or cooling obtained using a thermoelectric device depends its thermal efficiency. The maximum efficiency of a thermoelectric device for both power generation and cooling is determined by $=\frac{S 2 \sigma}{\kappa} T$

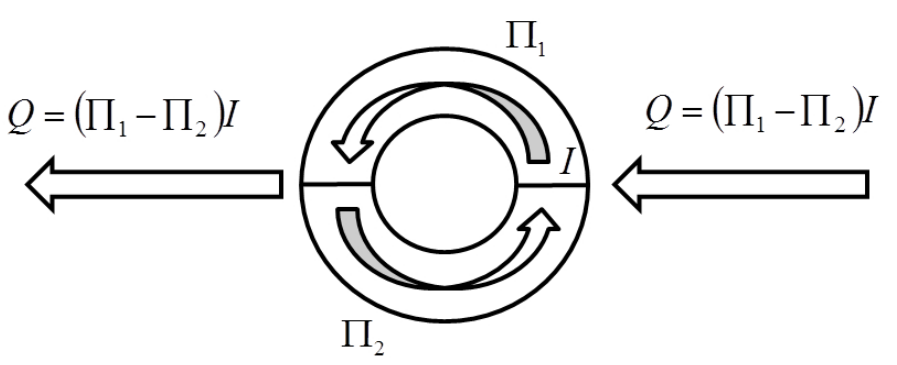

Figure 1. How Peltier effect takes place at junctions of two materials.

Thermoelectric materials have been studied recently in the forms of bulk thermoelectric materials, individual nanostructures, bulk nanostructures, and interfaces in bulk thermoelectric materials. Peltier heating and cooling happens when two materials with two different Peltier coefficients are joined together due to the imbalance of the Peltier heat owing in and out of junction. The cooling or heating, $Q$ happening at the junction is equal to

$Q=\left(\Pi_{2}-\Pi_{1}\right) I$

where, I is the electrical current, Peltier coefficient with subscripts 1 and 2 representing the two materials.

The total heat pumped by the device can be found from the number $(\mathrm{N})$ of junctions (pairs of N-type and P-type semi-conductors), the ratio of the length to area of these junctions (G) and the thermal conductance of the unit $(\kappa)$ for a given temperature distribution of the hot $\left(T_{H}\right)$ and cold $\left(T_{C}\right)$ faces, as a function of the supplied current [4];

$\dot{Q}_{C}=2 N\left[\alpha I T_{C}-\left(\frac{I^{2}}{2 G}\right)-\kappa\left(T_{H}-T_{C}\right) G\right]$

However, these equations can be simplified to obtain the linear resistance Seebeck co-efficient and conductance. These equations can be written after Luo and Bons (2008) as

$Q_{C}=-\kappa T_{H}+(\alpha I+\kappa) T_{C}-\frac{I^{2} R}{2}$

$\dot{Q}_{H}=\kappa T_{C}-(\alpha I-\kappa) T_{H}+\frac{I^{2} R}{2}$

$V=\alpha\left(T_{H}-T_{C}\right)+I R$ 
The resistance of the unit is available from manufacturer data (validated by manufacturer measurements). This helps derive the Seebeck co-efficient and the conductance [10].

$\alpha=\sqrt{\frac{2 R Q_{\max }}{T_{H}^{2}}}$

$\kappa=\frac{\alpha^{2}\left(T_{H}-\Delta T_{\max }\right)^{2}}{2 R \Delta T_{\max }}$

Coefficient of performance $(Z)$ and figure of merit $(z T)$ are also very useful in determining performance of thermoelectric devices according to Scherrer et.al. (2006)

\section{MODELLING AND SIMULATION}

\subsection{Mathematical Modelling}

The mathematical model above can be used in a simulation tool if the temperature and heat flux to be solved for the region. The values defined above can be used to obtain the Seeback coefficient, conductance and resistance as a function of temperature. The values of $\alpha, \kappa$ (derived using equations 5 and 6 above) and $R$ (obtained from the manufacturer data) were used in a commercially available CFD package Star $\mathrm{CCM}+$ to provide heat flux boundary conditions for the thermoelectric cooling model (TEC). Temperature boundary conditions were set for the hot side of the tool. The above system of six equations would be solved to obtain the heat flux and the temperature field. A simplified version of the boundaries defined is given in figure 2 .

In the simulation model in figure 2 above the well temperature is set to $160{ }^{\circ} \mathrm{C}, 180^{\circ} \mathrm{C}$ and $225^{\circ} \mathrm{C}$, respectively for two simulations.

Initial validation of the TECs were done using a linear interpolation and a curve fitting technique. Two test modules were considered in the analysis, one Bismuth Telluride (HT2) and the other custom hybrid bi-Te doped with lead (TESH127). In order to derive the linear equations for resistance and Seebeck co-efficiencts, data from the manufacturers were used. These values were verified with in house testing of the data. The so developed TEC model was valid in the tested temperature range. At elevated temperatures however, the performance could vary as the thermal conductance, electrical resistivity and Seebeck coefficient for the thermoelectric modules vary with temperature, each material having differing characteristics. In order to derive the linear equations that define the performance of the TECs at elevated temperature it is necessary to re-evaluate the manufacturer data values for these temperatures. In the case of the Laird module, it is possible to use the manufacturers analytical design tool, Aztec (Scillasoft, 2014) to find these values at the system temperature. When such data are not available, an alternative approach has to be followed. The resistance of the unit at a range of mean temperatures was measured in a laboratory oven and a digital multi meter to record to voltage drop across the unit at a fixed current. From these data, a linear equation for the resistance could be derived. In the absence of direct experimental data and extrapolation technique based on measurements and curve fitting was used to find values of $z T$ plots values for $z T$ at temperature for a range of materials. If the composition of the module was known, values could be estimated from these curves. In the absence of these values, the data from the experimental oven testing was used to approximate $z T$ with temperature. A copper heat sink was used to dissipate heat from the hot side, and the cold side was fixed to an insulated mass. Thermocouples were used to measure the hot and cold side temperatures.

Outer well fluid (at well temperature)

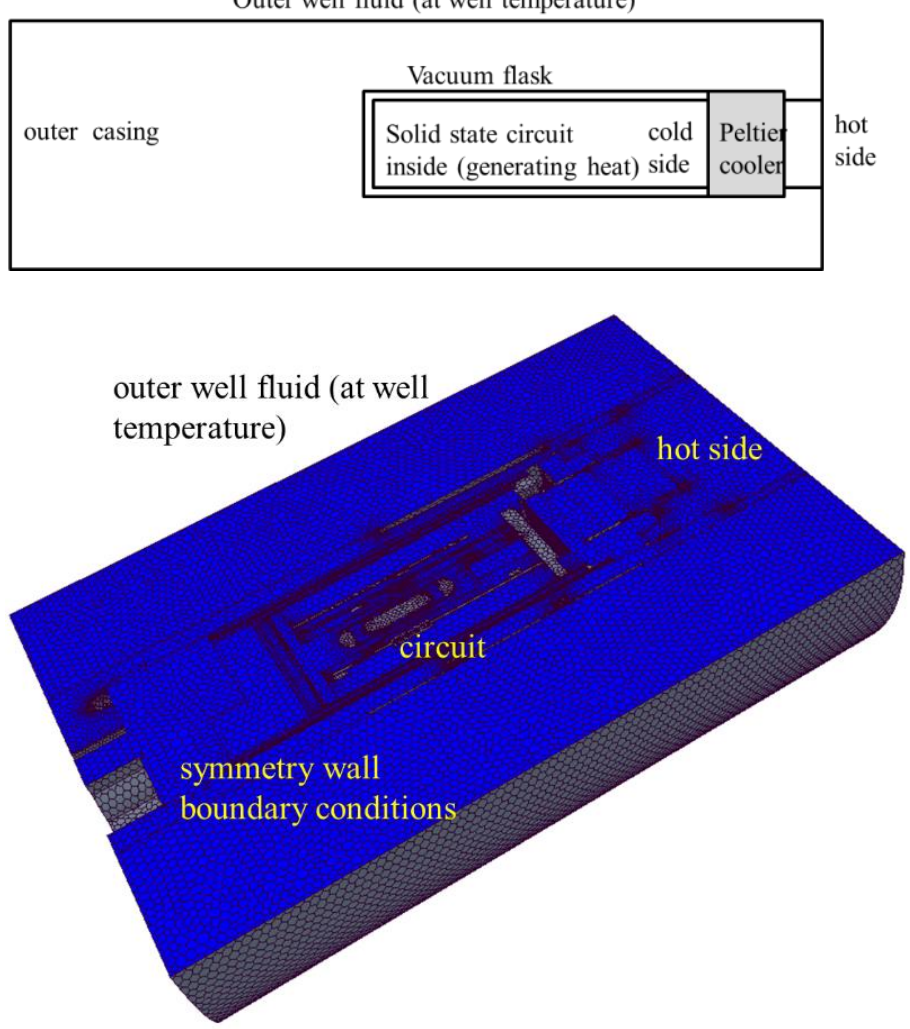

Figure 2. Boundaries and computational mesh.

The temperature performance has been predicted using derived linear equations [6]. Table 1 shows the values available from manufacturer data. These values are used in the CFD numerical tool in order to simulate the thermal performance. 


\section{MEASUREMENTS}

\subsection{CFD and Measurements}

The thermoelectric cooling performance was initially tested with a CFD cooling model of the Peltier device alone. Tests were carried out to characterize the TEC performance. The heat flux and cold side temperatures were measured at different hot side temperatures, viz: $160{ }^{\circ} \mathrm{C}, 180{ }^{\circ} \mathrm{C}$ and $225{ }^{\circ} \mathrm{C}$. The modules are fitted into an oven test rig, this comprises a copper heatsink and a vacuum flask. The system was placed in the oven at $160{ }^{\circ} \mathrm{C}, 180^{\circ} \mathrm{C}$ and $225^{\circ} \mathrm{C}$ and the time to heat the slug observed Figure 3 shows the temperature results of the TEC module using a linear interpolation technique. It shows that the predicted COP values are coherent with the measured and are able to predict the TEC performance satisfactorily. The tool modelled used for the purposes of the study was supplied by Avalon Sciences Ltd. It comprises a steel pressure barrel which houses the geophones, a mechanism to operate an arm which clamps the tool to the wall of the borehole and a module containing the digital electronics which perform the signal processing function. These electronics are housed within a vacuum insulated vessel. Active cooling is provided by a Thermoelectric Cooler (TEC) module similar to one that has been modelled above.

The numerical model is a three dimensional representation of the downhole tool similar to one that is commercially manufactured. To expedite the simulation, the regions adjacent to the digital electronics module are excluded from the model as there is no active components in this region and thus have no impact on the cooling of the electronics. To fully resolve all of the electronic components housed within the module would incur a high computational cost to accurately resolve the geometry and thus a simplified representation of the printed circuit board is used. This simplification does not hinder the performance analysis of heat transfer. The model takes advantage of the symmetry of the tool; only one half of the system is modelled, cut down the central axis of symmetry. (Figure 2) Planar symmetry conditions are applied to the cut faces. The external region of the model, representing the well fluid, has a fixed temperature boundary condition on the far face, representing the large thermal capacity of the borehole fluid.
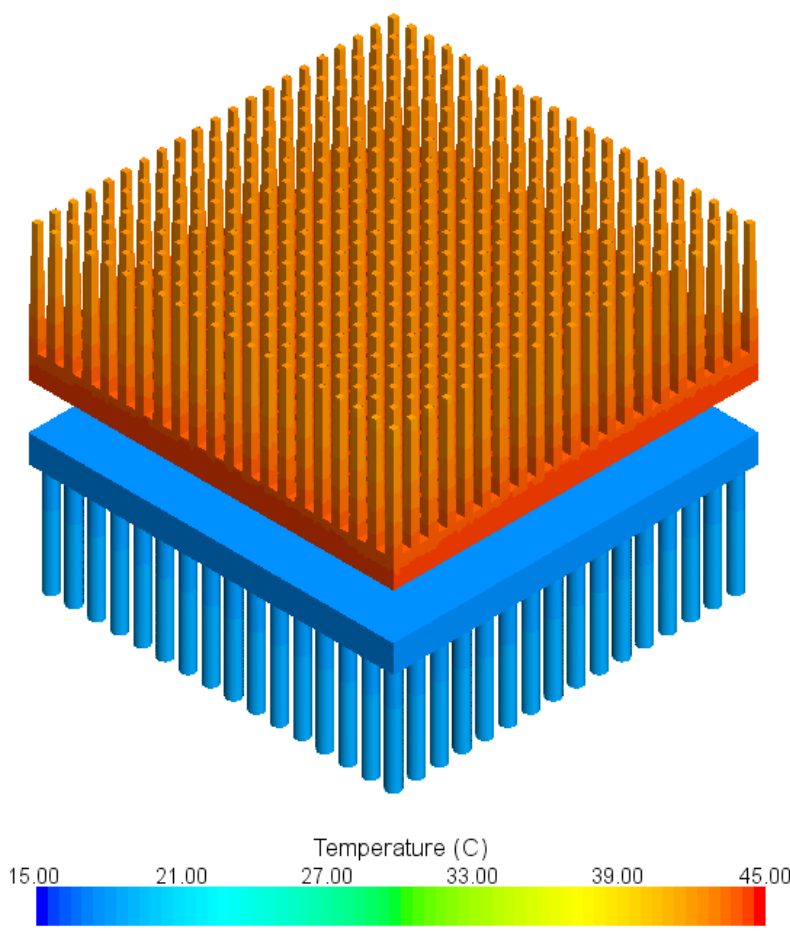

Figure 3. Simulation results for a typical thermoelectric module.

The fluid is modelled in the laminar regime, with convection driven by gravity in the direction that the tool is oriented in the well. The well fluid is modelled as water. The solid regions of the tool were modelled with appropriate material properties, sourced from the manufacturers' data sheet. The vacuum region of the flask is modelled as a gas with a conductivity of $1 \times 10^{-}$ ${ }^{6} \mathrm{Wm}^{-1} \mathrm{~K}^{-1}$.

\section{RESULTS}

Figures 4 shows the temperature profiles obtained with the computational simulation. The temperature profiles show that the cooling effect of the standard TEC based cooler. It is evident from the bright red colours in the heat sink side that the TEC performs well during cooling.

The main aim of the tool thermally is to isolate the hot fluid from the electronic circuitry and pump the heat generated out of the flask. Table 2 shows the fluid temperatures and flask temperatures with the cooling effect at a temperature difference.

A pumped hot oil bath was used to emulate the well fluid at elevated temperature and pressure. It is intended to take measurements in a well, but the controlled conditions in the laboratory represent the conditions down the hole. The limit of the fluid in the open system was $160{ }^{\circ} \mathrm{C}$. The tool was submersed in the fluid which was then heated. Once the system has reached steady state the temperature, as reported by a sensor embedded within the on-board electronics, 
was recorded. With the data from the experimental results at $160{ }^{\circ} \mathrm{C}$ external temperature the model was validated. The temperatures seen in the model are equivalent to those seen in the experimental testing.

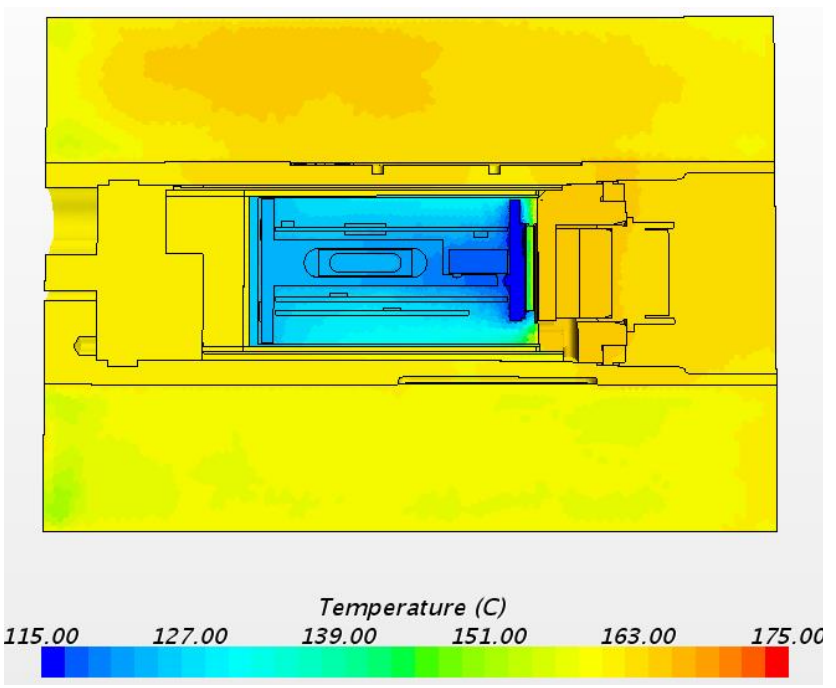

Figure 4. Temperature profile of the tool with the hybrid TEC module (TESH 127).

The results are in line with the results that were obtained with the simulation of the thermoelectric cooling device. The cooling effect of the TEC module is represented by the darker blue colour near the TEC of Figure 4.

Table 1. Summary of mean temperatures and cooling observed in experimental testing and simulation.

\begin{tabular}{lllll}
\hline & $\begin{array}{l}\text { Fluid } \\
\text { Temp. } \\
\left({ }^{\circ} \mathrm{C}\right)\end{array}$ & $\begin{array}{l}\text { Flask } \\
\text { Temp. } \\
\left({ }^{\circ} \mathrm{C}\right)\end{array}$ & $\begin{array}{l}\text { Cool- } \\
\text { ing } \\
\left({ }^{\circ} \mathrm{C}\right)\end{array}$ & $\begin{array}{l}\text { Volt- } \\
\text { age } \\
(\mathrm{V})\end{array}$ \\
\hline $\begin{array}{l}\text { Experi- } \\
\text { ment }\end{array}$ & 161.06 & 134.82 & 26.20 & 12.5 \\
HT2 & & & & \\
CFD & 160.00 & 133.77 & 26.20 & 11.6 \\
HT2 & & & & \\
\hline
\end{tabular}

The area just adjacent to the TEC unit shows a good cooling effect with a temperature outside the TEC in the model mid $190{ }^{\circ} \mathrm{C}$. This represents the heat pumping capacity of the TEC to the well fluid. This reduction is significant in the performance as it defines whether it leads to exceeding the threshold or not. The experimental results and the simulations show very good agreement as shown in table 1.

\section{CONCLUSIONS}

The main aim of the simulation work was to evaluate the performance of the downhole tool using TEC cooling module and then use the model as a base model in evaluating performance of similar tools. The numerical model was able to show the difference in performance within the tool geometry. The temperatures recorded in the electronic region of the module shows good agreement with the experimental values. However, the agreement on the voltage predictions is less impressive. This is, most likely due to the estimation of $\alpha$ value.

The first order linear approximation method gives realistic values for the TEC properties at elevated temperatures. This is more true for $Q$ and $T$. It is not yet confirmed how accurate it is for $\alpha$. A non-linear approximation method based on experimental values is under development at the moment and it could be more accurate at higher temperatures. Initial investigations have shown that the relationship is better explained with second and third order terms. This should give better predictions at elevated temperatures. However with current capabilities and available knowledgebase, the numerical model has been able to predict the thermal behaviour of the downhole measuring tool accurately. . This has lead to optimal designing of the tool geometry and TEC sizing. Accurate model prediction have also helped in making the customers understand about the tool geometry and cooling process. With a more advanced prediction models and further measurements, numerical models are expected to give accurate models that will eliminate the use of testing.

\section{ACKNOWLEDGMENTS}

The authors would like to thank Dr Tom Hughes for his contribution in the work and Avalon Sciences Ltd for the continual support for the project given as the industry partner.

The research work was funded by the knowledge transfer partnership grant No KTP009448 of Innovate UK.

\section{REFERENCES}

Bennett, G., Sherman, G. (1983), Analysis and thermal-design improvements of downhole tools for use in hot-dry wells http://www.osti.gov/pages/190 servlets/purl/6491119, doi:10.2172/6491119,

Shengqiang Bai, Hongliang Lu, Ting $\mathrm{Wu}$, Xianglin Yin, Xun Shi, and Lidong Chen. Numerical and experimental analysis for exhaust heat exchangers in automobile thermoelectric generators. Case Studies in Thermal Engineering, 4: 2014 99-112.

Dirker, J., Liu, W., Van Wyk, J.D., Meyer, J.P., and Malan, A.G.,( 2005) Embedded solid state heat extraction in integrated power electronic modules, Accepted for publication in the I.E.E.E. Transactions on Power Electronics, vol. 20, No. 3. 
Kim,W. et.al. (2006), Thermal conductivity reduction and thermoelectric figure of merit increase by embedding nanoparticles in crystalline semiconductors. Phys. Lett. Rev., 96,.

Kutasov, V. A., Lukyanova, L. N. and Vedernikov M. (2006), Thermoelectrics Handbook Macro to Nano. CRC, Boca Raton,. Ch. 37.

Luo, Z., (2008) A simple method to estimate the physical characteristics of a thermoelectric cooler. Electronics Cooling,.

Scherrer, H., and Scherrer, S.. Thermoelectrics Handbook Macro to Nano. CRC, Boca Raton, 2006. Ch. 27.

Sootsman, D.Y., Chung J.R. and Kanatzidis. M.G0(2009)., New and old concepts in thermoelectric materials. Angewandte Chemie (International ed.in English), 48:2009, 8616-8639.

Zhiting Tian, Sangyeop Lee, and Gang Chen. A comprehensive review of heat transfer in thermoelectric materials and devices. Ann. Rev. HeatTransfer, 17:2014, 425-483

Zhao, D., and Tan, G.. A (2014)review of thermoelectric cooling: Materials, modeling and applications. Applied Thermal Engineering, 66: No 1-2. 2014, 15-24, 